\title{
Electrophoretically Deposited Carbon Nanotube Spectrally Selective Solar Absorbers
}

\author{
Zhonghua Chena,b,*, Tobias Boström ${ }^{b}$ \\ a Norut Northern Research Institute Narvik AS, Narvik, Norway \\ a,b UiT, The Arctic University of Norway, Department of Physics and Technology, Troms $\varnothing$, Norway
}

\begin{abstract}
Three types of carbon nanotubes (CNT) have been investigated regarding their suitability as spectrally selective solar thermal absorbers. The CNT coatings were electrophoretically deposited on aluminum substrates using kinetically stable CNT aqueous suspensions, of which two CNT aqueous suspensions (N-CNT and P-CNT suspensions) were prepared as part of this study and the third one (T-CNT suspension) was purchased. The CNT suspension systems are simple and consist of only CNTs, DI water and a cathodic surfactant. Heat treated CNT coatings are visibly uniform. The CNT coating thickness, surface morphology and reflectance of CNT absorbers were characterized by White Light Interferometry, Scanning Electron Microscopy and Spectrophotometry, respectively. T-CNT absorber achieved a solar absorptance $\alpha$ of 0.79 and a thermal emittance $\varepsilon$ of 0.14 . N- and PCNT absorbers achieved better spectral selectivity, with $\alpha=0.90$ and $\varepsilon=0.14$ for N-CNT absorber, $\alpha=0.90$ and $\varepsilon=0.13$ for P-CNT absorber. The effect of CNT coating thickness, deposition parameters and peak heat treatment temperature on the spectral selectivity were studied. For these CNT suspension systems, the thickness and the heat treatment peak temperature are the key factors to achieving a good spectral selectivity.
\end{abstract}

Key-words: carbon nanotube, electrophoretic deposition, spectrally selective solar absorbers, solar absorptance, thermal emittance.

\section{Introduction}

Solar thermal absorbers are used to absorb and convert solar radiation into heat in solar thermal collectors. The solar absorber needs to be spectrally selective in order to be optically and thermally efficient, which translates to a high solar absorption in the UV-VIV-NIR solar spectrum and a low thermal emittance in the IR wavelength region. They are usually constructed with a selectively solar absorbing thin film coated on a highly infrared reflective metal substrate. The top absorbing layer is designed to absorb the solar radiation while the metal substrate should reflect infrared light, i.e. heat. Solar absorbers having this structure are called tandem absorbers [1].

Highly reflective metals such as aluminum [2-4], copper [5] and stainless steel [6] are commonly used as substrates. For the absorbing layer, electroplated black chrome [7, 8], black nickel [9, 10], and cermets [11-13] are among the most used materials. Techniques like anodization, electroplating, sputtering, spin-coating, chemical and physical vapor deposition are or have been utilized to fabricate absorbing coatings. Most manufacturing methods require costly high technology equipment, high energy consumption or toxic chemicals, and are therefore not environment-friendly. In this study, direct current electrophoretic deposition (EPD) has been employed to prepare absorbing coatings on aluminum substrates. EPD can be applied with simple devices and uses simple systems like aqueous suspensions. The chemical consumption is very low and the deposition process takes a few seconds at ambient conditions. Electrophoretically deposited coatings exhibit good microstructure homogeneity and the

*Corresponding author. Tel.: +47 76965350.

E-mail address: zhonghua.chen@ norut.no (Z. Chen) 
thickness of coating can be easily controlled by tuning the EPD parameters, such as voltage, inter-electrode spacing, and deposition time [14].

Carbon nanotube (CNT) was discovered by Iijima in 1991 [15]. Since then, it has been extensively studied due to their unique thermal, electronic and mechanical properties. Aligned CNTs array also has good light absorptance at wavelengths from far ultraviolet to near infrared [16]. A tandem structure prepared by synthesizing aligned CNTs on Au films didn't exhibit spectral selectivity over the solar spectrum though a high absorptance of $95 \%$ was attained [17]. However, our previous work simulating solar absorber using single-walled carbon nanotube coating as absorbing layer indicated that CNT absorber exhibited good spectral selectivity [18]. The objective of this work has been to experimentally investigate the suitability of electrophoretically deposited CNT coatings on aluminum substrates for use as spectral selective solar absorbers.

\section{Methods}

Three types of CNTs were investigated: T-CNT, N-CNT and P-CNT. T-CNT aqueous suspension was provided by the company n-Tec AS and consisted of functionalized multi-walled CNTs, water and a cathodic surfactant. The suspension was sonicated in a bath for 2 hours before EPD. P-CNT and N-CNT are also functionalized multiwalled CNTs and were obtained from the companies Plasmachem and Nanocyl, respectively. These two types of as-purchased CNTs were used to prepare CNT aqueous suspensions without any pre-treatments. To prepare NCNT suspension, a cathodic surfactant was first dissolved in DI water with assistance of stir before adding NCNTs. Then the mixture was subjected to a sonication bath for dispersion until a stable CNT suspension was formed. The P-CNT suspension was prepared using the same procedure. All the three suspensions had a solid content of $0.1 \mathrm{wt} \%$. The kinetically stable suspensions can be stored for months with insignificant sedimentation.

EPD is a facile process for fabrication of films and coatings from aqueous or organic suspensions on substrates and bulk bodies with even complicated surfaces. Under an applied electric field, charged particles in the suspension move toward and deposit on the substrate with the opposite charge. In CNT suspensions, CNTs stabilized by the existed cathodic surfactant are negative charged. Therefore, the CNT coating is deposited on the anode. Fig.1 is a schematic diagram of electrophoretic deposition of carbon nanotubes. Highly specularly reflecting aluminum were used as electrodes i.e. substrates. The deposited area is $30 \mathrm{~mm} \times 27 \mathrm{~mm}$. The EPD process takes only a few seconds. As-deposited CNT coating is wet and has a bad adhesion to the $\mathrm{Al}$ substrate. Following drying and heat treatment are necessary to solidify the CNT coating. The samples can be heat treated directly after EPD, though, in this work all samples were dried for 20 hours at ambient conditions before heat treatment. Then they were heat treated in a tube furnace under an atmosphere environment. The rate of temperature increase was set to $50{ }^{\circ} \mathrm{C}$ per minute. The peak/final temperature $T_{\mathrm{p}}$ was tuned for different purposes. The dwell time at $T_{\mathrm{p}}$ was fixed to $5 \min$ based on the results of previous experiments. Then the CNT absorber samples were left in the tube furnace until the temperature decreased to $300{ }^{\circ} \mathrm{C}$ before they were removed and stored at room temperature for fast cooling. The heat treated CNT coatings have a fairly well adhesion to the aluminum substrates and are resistant to taping.

There are a few EPD factors such as CNT concentration, electric field, inter-electrode spacing, deposition time affecting the quality and performance of the CNT coatings. The deposition mass i.e. the coating thickness for planar geometries is proportional to the solid concentration, the electrophoretic mobility, the electric field and the deposition time [19]. For an aqueous CNT suspension system, the electrophoretic mobility of CNTs is related to the CNT concentration. To simplify the experiments in this study, the effect of CNT coating thickness was investigated by tuning only the deposition time while the CNT concentration for the respective CNT type was kept constant throughout all experiments. In addition, the effect of the applied voltage while keeping a constant electric field and the effect of peak temperature of the heat treatment were also investigated. 


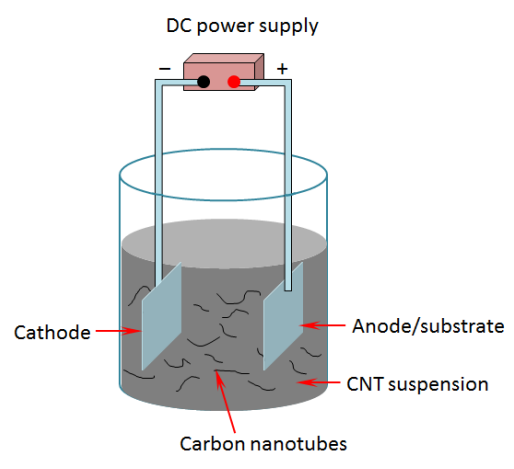

Fig. 1: Schematic diagram of electrophoretic deposition of carbon nanotubes.

\section{Characterization}

\subsection{Characterization tools}

During EPD, aluminum substrate was taped partly, which created a step where the thickness can be measured. A Veeco NT9080 Profilometer which uses high precision white light interferometry was employed for thickness measurement after heat treatment. Surface morphology and atomic composition of the samples were investigated by a ZEISS Merlin VP Scanning Electron Microscope equipped with an EDS detector from Oxford Instruments. Reflectance in the wavelength interval 0.3-2.5 $\mu \mathrm{m}$ was measured with a Perkin-Elmer Lambda 900 spectrophotometer equipped with an integrating sphere of $150 \mathrm{~mm}$ diameter, circular beam entrance and sample port of $25 \mathrm{~mm}$. A Bruker Tensor27 FT-IR spectrophotometer was employed to measure the reflectance in the infrared wavelength range $2.5-20 \mu \mathrm{m}$. The obtained reflectance data were used to calculate solar absorptance and thermal emittance of solar absorbers as introduced in section 3.2.+

\subsection{Optical characterization}

A SSA is usually evaluated by two parameters - the solar absorptance and the thermal emittance under normal incidence of radiation. Normal solar absorptance, $\alpha$, is defined as a weighted fraction of the absorbed radiation to the incoming solar radiation on a surface (Eq. (1)).

$$
\alpha=\frac{\int_{0.3}^{2.5} I_{s o l}(\lambda)(1-R(\lambda)) d \lambda}{\int_{0.3}^{2.5} I_{s o l}(\lambda) d \lambda}
$$

The spectral solar irradiance, $I_{\mathrm{sol}}$, is defined according to ISO standard 9845-1 (1992) for air mass of 1.5. $\lambda$ is the wavelength of incident radiation in units of $\mu \mathrm{m}$ and $R(\lambda)$ is the reflectance at a certain wavelength.

Normal thermal emittance, $\varepsilon$, is the ratio of emitted radiation of a surface to that of Plank's blackbody, $I_{\mathrm{p}}$, at $100{ }^{\circ} \mathrm{C}$ and can be calculated following

$$
\varepsilon=\frac{\int_{2.0}^{50} I_{p}(\lambda)(1-R(\lambda)) d \lambda}{\int_{2.0}^{50} I_{p}(\lambda) d \lambda}
$$

Because the reflectance data only could be measured up to $20 \mu \mathrm{m}$, an extrapolation method was introduced to estimate the reflectance from 20 to $50 \mu \mathrm{m}$ so that the calculation by Eq. (2) can be completed. The standard deviation of $\alpha$ and $\varepsilon$ is respectively 0.002 and 0.01 for the solar absorber samples in this study. The standard deviation has been calculated from repeated reflectance measurements of the same sample over an extended time period. 
There are several ways to evaluate the spectral selectivity. One of the most frequently used methods is to calculate the ratio of solar absorptance to thermal emittance $(\alpha / \varepsilon)$. However, we judge this method to be inappropriate to assess photothermal conversion efficiency. For example, a solar absorber with a solar absorptance of 0.60 and a thermal emittance of 0.03 achieves a ratio of 20 but doesn't have higher photothermal conversion efficiency than an absorber with a solar absorptance of 0.90 and a thermal emittance of 0.1 which achieves $\alpha / \varepsilon$ of only 9. The opposite is actually true since the solar absorptance is twice as important as the thermal emittance. In order to rate the spectral selectivity and hence the performance of a solar absorber we use the expression $\alpha-0.5 \varepsilon$ in this study to reflect the weight factor of thermal emittance in a more reasonable matter. This expression was first recommended in a report of the IEA solar heating and cooling program Task 27 [20], and has been employed in the newly issued international standard ISO 22975-3: absorber surface durability [21].

\section{Results and Discussion}

\subsection{Surface morphology}

Surface morphology of the three types of CNT absorbers and the respective shape of CNTs can be seen in Fig. 2. For T-CNT absorber (Fig. 2(a)), the CNTs are stretched and mostly parallel to the aluminum substrate. The CNT coating is embedded with carbon impurities and the surfactant. The CNTs of N-CNT absorber (Fig. 2(b)) and P-CNT (Fig. 2(c)) are curved and nested like cotton, resulting in better light trapping i.e. higher absorption of solar radiation. The black spots appearing in the SEM images originate from the aluminum substrate. Compared to N-CNT and P-CNT absorbers, T-CNT absorbers have a lower density of CNT, hence a rougher surface and a lower coverage of the substrate. This can also be evidenced by the interband absorption of aluminum at $800 \mathrm{~nm}$ [22] as indicated in Fig. 3(a). The lower density of CNT might be due to a lower purification from original asproduced CNTs or higher surfactant concentration in CNT suspension. The stripe pattern originating from the aluminum substrate is more clearly seen in the SEM images of N-CNT and P-CNT absorbers (Fig. 2(b) and 2(c)), indicating a smoother surface which can be confirmed by the value of roughness $R_{\mathrm{a}}$ measured by the Profilometer. $R_{\mathrm{a}}$ is about $0.2 \mu \mathrm{m}$ for T-CNT absorber and only $0.03 \mu \mathrm{m}$ for both N- and P-CNT absorbers.

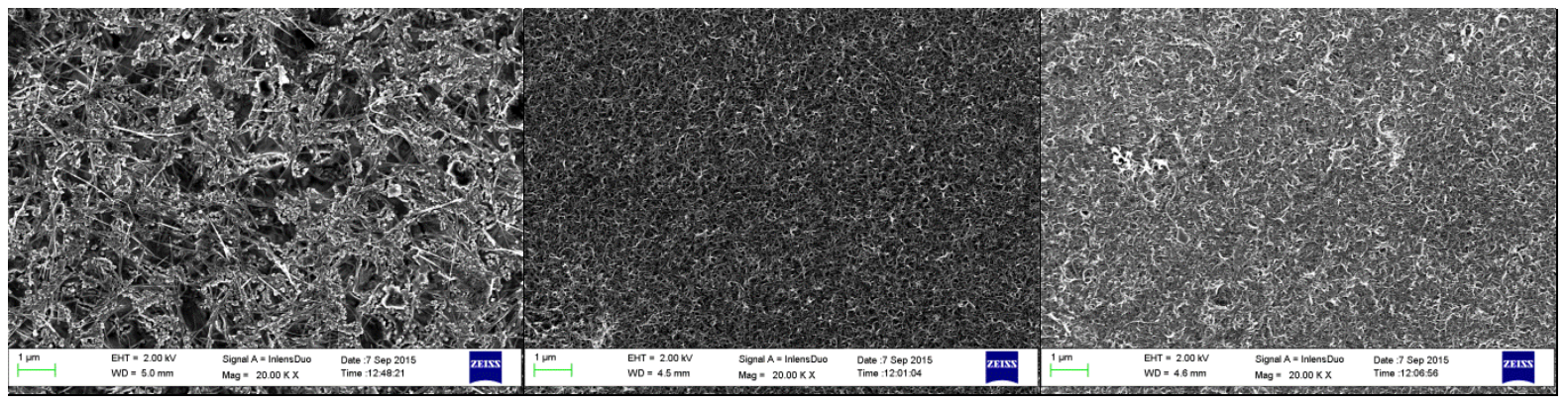

(a)

(b)

(c)

Fig. 2: SEM images of CNT absorbers under a magnification of 10K, (a) T-CNT absorber; (b) N-CNT absorber; (c) P-CNT absorber.

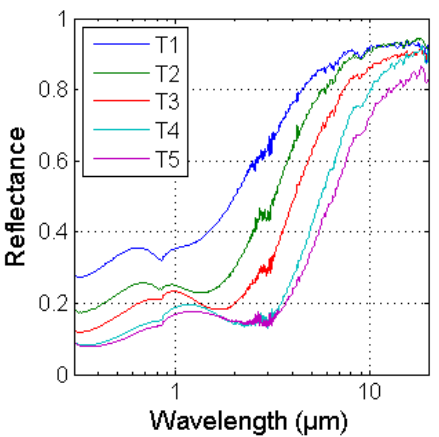

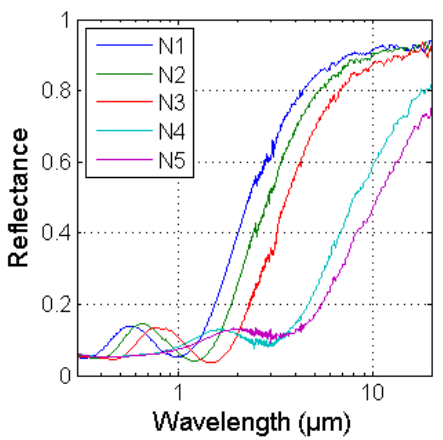

b

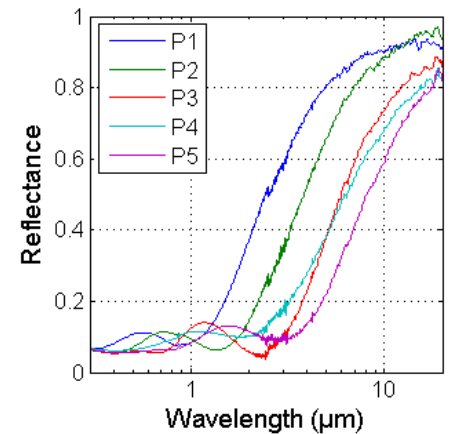

Fig. 3: Reflectance of CNT absorbers: a) T-CNT absorbers; b) N-CNT absorbers; c) P-CNT absorbers. 


\subsection{Effect of coating thickness}

Different thicknesses of CNT coatings were deposited by varying the deposition time while keeping an applied voltage of $20 \mathrm{~V}$ and an inter-electrode spacing of $10 \mathrm{~mm}$. After drying, the samples were heat treated with a peak temperature of $500{ }^{\circ} \mathrm{C}$. The dwell time at peak temperature was $5 \mathrm{~min}$. The reflectance spectrum for each type of CNT is shown in Fig. 3.

The CNT coating thickness increases from sample T1 to T5 (Fig. 3(a)), from N1 to N5 (Fig. 3(b)) and from P1 to P5 (Fig. 3(c)). The effect of CNT coating thickness on reflectance is quite similar for all the three types of CNTs: with an increasing thickness, the reflectance values in both the visible and infrared range are reduced, and the transition from low to high reflectance is shifted to longer wavelengths. As a result, both the solar absorptance and the thermal emittance increase with an increasing film thickness, as showed in Tab. 1. The increase of solar absorptance is desired which improves the spectral selectivity, while higher thermal emittance downgrades the spectral selectivity. Consequently, there exists, in terms of spectral selectivity, an optimum film thickness for each CNT type, see Fig. 4.

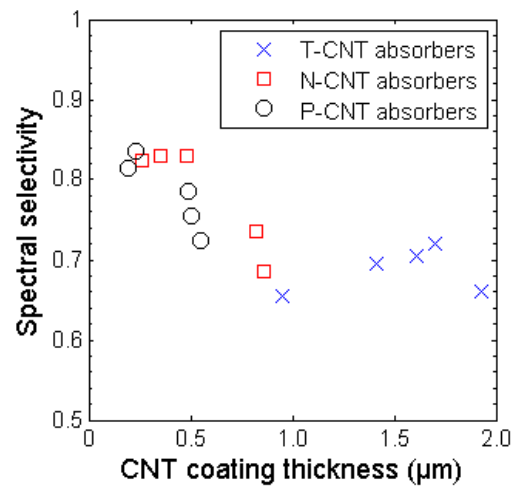

Fig. 4: Spectral selectivity of CNT absorbers vs CNT coating thickness.

For T-CNT absorbers, the best achieved spectral selectivity was 0.72 for the samples heat treated at a peak temperature of $500{ }^{\circ} \mathrm{C}$, of which the solar absorptance was 0.87 and the thermal emittance was 0.30 . The measured thickness of CNT coating at the optimum spectral selectivity is $1.7 \mu \mathrm{m}$. The optimum thickness for N-CNT and PCNT was $0.48 \mu \mathrm{m}$ and $0.23 \mu \mathrm{m}$ respectively. The CNT coating is much thicker for the T-CNT absorber with the best spectral selectivity. It can be partly explained by the lower CNT density indicated in the SEM image. The higher CNT density for N-CNT and P-CNT absorbers enhances the light absorption. Another key factor in explaining this difference in the optimal thickness is that the T-CNT absorber has a much rougher surface compared to N- and P-CNT absorbers as indicated in section 4.1, and does hence not show a pronounced interference pattern. Consequently, it cannot make use of thin film interference and only relies on intrinsic absorption of light. Therefore, it must be much thicker in order to get a high solar absorption but then on the expense of an unwanted higher thermal emittance. In order to be able to produce an efficient spectrally selective solar absorber, the sharp transition from low to high reflectance should occur in the wavelength range around 2 $\mu \mathrm{m}$. This can only be achieved by the combined effect of intrinsic absorption and importantly also the thin film interference effect. N- and P-CNT absorbers exhibit a much smoother surface and can thereby utilize the thin film interference effect to a much greater extent. The solar absorption and the selectivity can be enhanced by optimizing the film thickness so that the first destructive interference minimum is placed at about 1-2 $\mu \mathrm{m}$, see N2/N3 and P2 in Fig.3.

N-CNT absorbers obtained a spectral selectivity of 0.83 , based on a sample with a solar absorptance of 0.90 and a thermal emittance of 0.14 , while P-CNT absorbers achieved 0.84 with a solar absorptance of 0.90 and a thermal emittance of 0.13 . It is worth noting that the results of the thickness measurement have a higher uncertainty for thinner coatings due to the inherent surface roughness of the aluminum substrates. 
Tab. 1: The effect of CNT coating thickness on solar absorptance and thermal emittance.

\begin{tabular}{|c|c|c|c|c|c|c|c|c|c|c|c|}
\hline $\begin{array}{c}\text { T-CNT } \\
\text { Samples }\end{array}$ & $\begin{array}{c}\text { Coating } \\
\text { thickness } \\
(\mu \mathrm{m})\end{array}$ & $\alpha$ & $\varepsilon$ & $\begin{array}{l}\text { N-CNT } \\
\text { Samples }\end{array}$ & $\begin{array}{c}\text { Coating } \\
\text { thickness } \\
(\mu \mathrm{m})\end{array}$ & $\alpha$ & $\varepsilon$ & $\begin{array}{c}\text { P-CNT } \\
\text { Samples }\end{array}$ & $\begin{array}{c}\text { Coating } \\
\text { thickness } \\
(\mu \mathrm{m})\end{array}$ & $\alpha$ & $\varepsilon$ \\
\hline $\mathrm{T} 1$ & 0.95 & 0.71 & 0.11 & N1 & 0.26 & 0.87 & 0.09 & $\mathrm{P} 1$ & 0.19 & 0.87 & 0.11 \\
\hline $\mathrm{T} 2$ & 1.41 & 0.79 & 0.19 & $\mathrm{~N} 2$ & 0.35 & 0.89 & 0.12 & $\mathrm{P} 2$ & 0.23 & 0.90 & 0.13 \\
\hline $\mathrm{T} 3$ & 1.61 & 0.85 & 0.29 & N3 & 0.48 & 0.90 & 0.14 & P3 & 0.49 & 0.92 & 0.27 \\
\hline $\mathrm{T} 4$ & 1.70 & 0.87 & 0.30 & N4 & 0.82 & 0.93 & 0.39 & P4 & 0.50 & 0.91 & 0.31 \\
\hline T5 & 1.93 & 0.91 & 0.50 & N5 & 0.86 & 0.93 & 0.49 & P5 & 0.55 & 0.92 & 0.39 \\
\hline
\end{tabular}

\subsection{Effect of deposition parameters}

The electrical field intensity influences the resulting deposition thickness significantly, it is determined by the applied voltage and the spacing between the two electrodes. The same electrical field intensity can be achieved by varying voltage and spacing. An investigation on whether or not this variation has an effect on the reflectance and resulting selectivity of the CNT absorbers has been done only for N-CNT and P-CNT absorbers since they are more promising candidates than T-CNT absorbers. Based on our previous study [23], an electrical field intensity of $2 \mathrm{~V} / \mathrm{mm}$ was chosen and kept for this investigation. The voltage was set to 20, 30 and $40 \mathrm{~V}$. Correspondingly, the spacing was adjusted to 10, 15 and $20 \mathrm{~mm}$. Except for the applied voltage and spacing, the other parameters were kept the same for EPD of each type of CNT samples. The results of solar absorptance and thermal emittance are listed in Tab. 2. The voltage and spacing has little impact on P-CNT absorbers but some on the emittance of the N-CNT absorbers.

Tab. 2: The effect of electrophoretic voltage on solar absorptance and thermal emittance.

\begin{tabular}{|c|c|c|c|c|c|c|}
\hline \multirow{2}{*}{ Electric field (V/mm) } & \multirow{2}{*}{ Voltage (V) } & \multirow{2}{*}{ Spacing $(\mathrm{mm})$} & \multicolumn{2}{|c|}{ N-CNT } & \multicolumn{2}{|c|}{ P-CNT } \\
\hline & & & $\alpha$ & $\varepsilon$ & $\alpha$ & $\varepsilon$ \\
\hline 2 & 20 & 10 & 0.91 & 0.18 & 0.90 & 0.20 \\
\hline 2 & 30 & 15 & 0.90 & 0.14 & 0.90 & 0.20 \\
\hline 2 & 40 & 20 & 0.91 & 0.20 & 0.90 & 0.21 \\
\hline
\end{tabular}

\subsection{Effect of peak temperature in heat treatment}

To investigate the effect of peak temperature during heat treatment, a set of CNT absorber samples were deposited and divided into 4 groups which were heat treated with different peak temperatures $-300{ }^{\circ} \mathrm{C}, 400{ }^{\circ} \mathrm{C}$, $500{ }^{\circ} \mathrm{C}$ and $600{ }^{\circ} \mathrm{C}$. The samples based on the same type of CNT used identical parameters for electrophoretic deposition. All the samples were heat treated after 20 hours of drying at room temperature. A temperature increase rate of $50{ }^{\circ} \mathrm{C} / \mathrm{min}$ and a dwell time of $5 \mathrm{~min}$ at peak temperature were kept for all the samples. Different from the samples in other sections, the samples in this section were left in the furnace until the temperature had decreased to $250{ }^{\circ} \mathrm{C}$ before they were taken out and cooled at room temperature. 


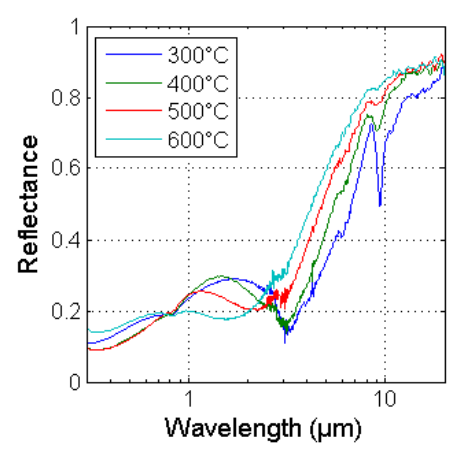

(a)

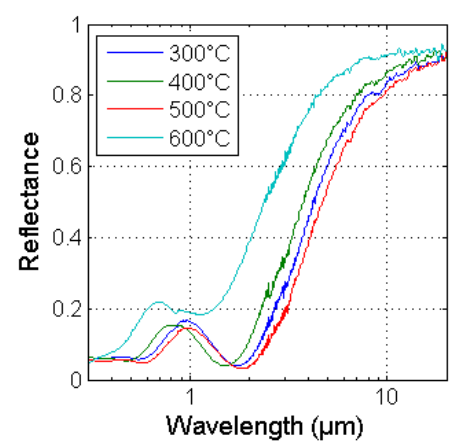

(b)

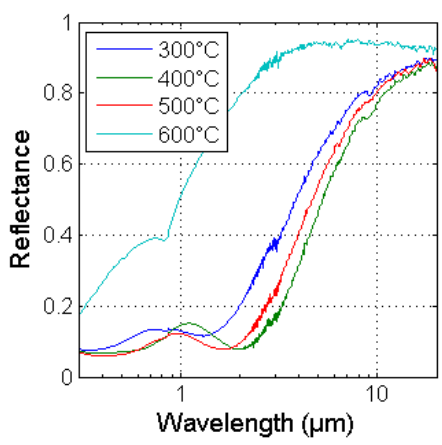

(c)

Fig. 5: Reflectance at different peak temperatures, (a) T-CNT absorbers; (b) N-CNT absorbers; (c) P-CNT absorbers.

Tab. 3: The effect of peak temperature on solar absorptance and thermal emittance.

\begin{tabular}{|c|c|c|c|c|c|c|c|c|c|}
\hline $\begin{array}{c}\text { Peak T } \\
\left({ }^{\circ} \mathbf{C}\right)\end{array}$ & $\begin{array}{c}\text { T-CNT } \\
\text { Samples }\end{array}$ & $\boldsymbol{\alpha}$ & $\boldsymbol{\varepsilon}$ & $\begin{array}{c}\text { N-CNT } \\
\text { Samples }\end{array}$ & $\boldsymbol{\alpha}$ & $\boldsymbol{\varepsilon}$ & $\begin{array}{c}\text { P-CNT } \\
\text { Samples }\end{array}$ & $\boldsymbol{\alpha}$ & $\boldsymbol{\varepsilon}$ \\
\hline 300 & $\mathrm{~T} 6$ & 0.79 & 0.31 & $\mathrm{~N} 6$ & 0.90 & 0.19 & $\mathrm{P} 6$ & 0.87 & 0.18 \\
400 & $\mathrm{~T} 7$ & 0.80 & 0.22 & $\mathrm{~N} 7$ & 0.89 & 0.16 & $\mathrm{P} 7$ & 0.90 & 0.24 \\
500 & $\mathrm{~T} 8$ & 0.81 & 0.19 & $\mathrm{~N} 8$ & 0.91 & 0.20 & $\mathrm{P} 8$ & 0.91 & 0.21 \\
600 & $\mathrm{~T} 9$ & 0.79 & 0.14 & $\mathrm{~N} 9$ & 0.80 & 0.10 & $\mathrm{P} 9$ & 0.54 & 0.06 \\
\hline
\end{tabular}

The reflectance of each type of CNT absorbers is shown in Fig. 5, the solar absorptance and the thermal emittance are listed Tab. 3. For T-CNT absorbers, a higher peak temperature during heat treatment reduces the thermal emittance while the solar absorptance changes little. This results in an improvement on the overall spectral selectivity. For a peak temperature of $300{ }^{\circ} \mathrm{C}, \mathrm{T}-\mathrm{CNT}$ absorber has a spectral selectivity of 0.64 and it increases to 0.72 for $600{ }^{\circ} \mathrm{C}$, as shown in Fig. 6. This behavior could be explained by incineration of carbon impurities other than CNTs and the surfactant. With higher temperature, the samples are also heat treated longer. Both factors result in a thinner coating that reduces the thermal emittance. There is no use in increasing the peak temperature further since the aluminum substrate has a melting point of $660{ }^{\circ} \mathrm{C}$. The solar absorptance and the thermal emittance of $\mathrm{N}$ - and P-CNT varied with different peak temperatures from $300{ }^{\circ} \mathrm{C}$ to $500{ }^{\circ} \mathrm{C}$, although the spectral selectivity was little affected (Fig. 6). For N- and P-CNT absorbers heat treated at $600{ }^{\circ} \mathrm{C}$, the CNT coatings are seriously damaged compared to T-CNT absorbers. This can be seen in the digital photos of these samples, shown in Fig. 7. The lower thermal stability of N-and P-CNTs is probably due to the induced structural defects on the outer walls of CNTs and the shortening of CNTs during the functionalization process by acids [24-25]. The damage due to charring of CNTs starts at temperatures over $500{ }^{\circ} \mathrm{C}$. It results in a dramatic drop in the solar absorptance. With a peak temperature during heat treatment of $500{ }^{\circ} \mathrm{C}$, the N-CNT absorber achieves a spectral selectivity of 0.82 while it is 0.81 for P-CNT absorber.

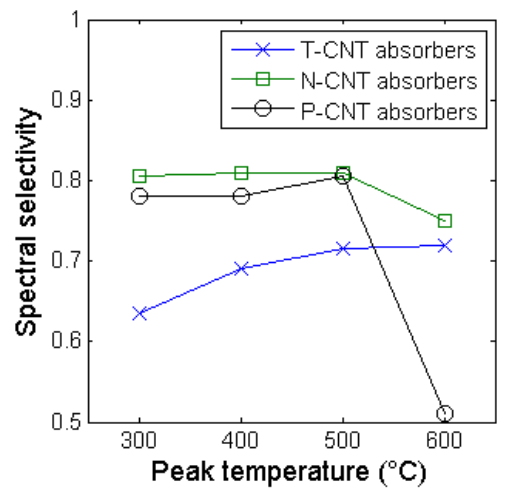

Fig. 6: Spectral selectivity of CNT absorbers at different peak temperatures. 


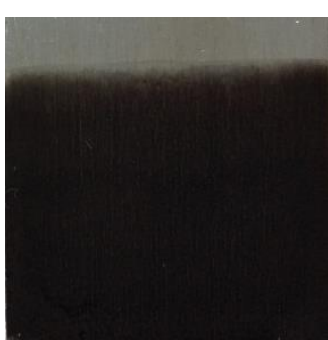

(a)

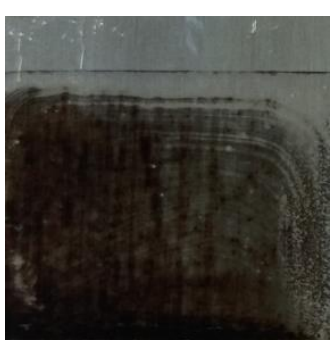

(b)

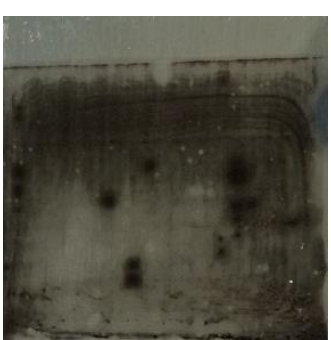

(c)

Fig. 7: CNT absorbers heat treated at $600{ }^{\circ} \mathrm{C}$, (a) T-CNT absorber; (b) N-CNT absorber; (c) P-CNT absorber.

\section{Conclusions}

Functionalized carbon nanotubes (CNT) from different production methods were successfully deposited on aluminum substrates by electrophoresis. We demonstrated that Electrophoretic Deposition (EPD) is a fast, cheap and facile method for preparing CNT coatings. Compared to the traditional processes of fabricating spectrally selective absorbers, EPD is very fast and is more environment-friendly owing to the used aqueous suspensions and the low chemical consumption. In addition, the production process does not require any advanced coating equipment and the absorbers only need to be heat treated in air, therefore no need for an inert atmosphere. The Nand P-CNT absorbers exhibit good spectral selectivity, which is over 0.80 at optimal conditions. With thicker CNT coating, the transition from low to high reflectance of the CNT absorbers shifts to longer wavelengths, which means a large gain in solar absorptance and reduced thermal emittance. The EPD parameters play an important role in effecting the spectral selectivity of CNT absorbers to different extents. Higher peak temperatures up to $500{ }^{\circ} \mathrm{C}$ in the heat treatments improved the spectral selectivity of the CNT absorbers. However, at a peak temperature of $600{ }^{\circ} \mathrm{C}$, the $\mathrm{N}$ - and P-CNTs were charred or evacuated, with the CNT coatings destroyed or nonuniform. The best performance achieved was a solar absorptance of 0.90 and a thermal emittance of 0.13 which yielded a selectivity of 0.84 , which is normally not commercially competitive. However, since the production method is fast, simple and inexpensive, cheap absorbers with a slight lower selectivity could still be of commercial interests. Further work will assess the durability of the CNT absorbers by subjecting them to high temperature and condensation accelerated ageing tests.

\section{Acknowledgements}

This work was supported by the Nano2021 program of The Research Council of Norway, project number 219161 and the company ASV Solar AS.

\section{References}

[1] Agnihotri, O.P., Gupta, B.K., 1981. Solar Selective Surfaces. John Wiley \& Sons, New York.

[2] T. Boström, G. Westin, E. Wäckelgård. Solution-chemical derived nickel-alumina coatings for thermal solar absorbers. Solar Energy 74 (2003) 497-503.

[3] S. S. Tinchev, P. I. Nikolova, Y. T. Dyulgerska, Thermal solar absorber made of diamond-like carbon thin films. Journal of Physics: Conference Series 223 (2010) 012017.

[4] P. Konttinen, T. Salo, P.D. Lund, Degradation of unglazed rough graphite-aluminium solar absorber surfaces in simulated acid and neutral rain. Solar Energy78 (2005) 41-48.

[5] X. Xiao, L. Miao, G. Xu, L. Lu, Z. Su, N. Wang, S. Tanemura, A facile process to prepare copper oxide thin films as solar selective absorbers. Applied Surface Science 257 (2011) 10729-10736.

[6] M. Joly, Y. Antonetti, M, Python, M. Gonzalez, T. Gascou, J. L. Scartezzini, A. Schüler, Novel black selective coating for tubular solar absorbers based on a sol-gel method. Solar Energy 94 (2013) 233-239.

[7] K. Shanker, P. H. Holloway. Electrodeposition of black chrome selective solar absorber coatings with improved thermal stability. Thin Solid Films, 127 (1985) 181-189. 
[8] R.L. Axelbaum, H. Brandt. The effect of substrate surface preparation on the optical properties of a black chrome solar absorber coating. Solar Energy Vol. 39 No. 3, pp. 233-241, 1987.

[9] K. N. Srinivasan, N.V. Shanmugam, M. Selvam, S. John, B. A. Shenoi. Nickel-black solar absorber coatings. Energy Convers. Mgmt vol. 24, No. 4 pp. 255-258, 1984.

[10] M. Lira-Cantu', A. M. Sabio, A. Brustenga, P. Go'mez-Romero. Electrochemical deposition of black nickel solar absorber coatings on stainless steel AISI316L for thermal solar cells. Solar Energy Materials and Solar Cells 87 (2005) 685-694.

[11] L. Zheng, F. Zhou, Z. Zhou, X. Song, G. Dong, M. Wang, X. Diao. Angular solar absorptance and thermal stability of Mo-SiO2 double cermet solar selective absorber coating. Solar Energy 115 (2015) 341-346

[12] L. Rebouta, A. Sousa, P. Capela, M. Andritschky, P. Santilli, A. Matilainen, K. Pischow, N.P. Barradas, E. Alves. Solar selective absorbers based on Al2O3:W cermets and AlSiN/AlSiON layers. Solar Energy Materials and Solar Cells 137 (2015) 93-100.

[13] T. Boström, G. Westin and E. Wäckelgård. Optimization of a solution-chemically derived solar absorbing spectrally selective surface. Solar Energy Materials and Solar Cells, 91 (2007) 38-43.

[14] A.R. Boccaccini, I. Zhitomirsky, 2002. Application of electrophoretic and electrolytic deposition techniques in ceramics processing. Current Opin Solid State Mater Sci. 6, 251-60.

[15] Iijima, S., 1991. Helical microtubules of graphitic carbon. Nature. 354, 56-58.

[16] X. Wu, L. Pan, H. Li, X. Fan, T.Y.Ng, D. Xu. 2003. Optical properties of aligned carbon nanotubes. Phys. Rev. B. 68, 193401.

[17] A. Cao, X. Zhang, C. Xu, B. Wei, D. Wu. Tandem structure of aligned carbon nanotubes on Au and its solar thermal absorption. Solar Energy Materials and Solar Cells. 70 (2002) 481-486.

[18] Z. Chen, A. Jain, T. Boström, Simulation of Anti-reflection coated carbonaceous spectrally selective absorbers. Energy Procedia 58 ( 2014 ) 179-184.

[19] D. Hanaor, M. Michelazzi, P. Veronesi, C. Leonelli, M. Romagnoli, C. Sorrell. Anodic Aqueous Electrophoretic Deposition of Titanium Dioxide Using Carboxylic Acids as Dispersing Agents. Journal of the European Ceramic Society, 31(6), 1041-1047, 2011

[20] B. Carlsson. Recommended qualification test procedure for solar absorber surface durability. IEA SHC Task 27 draft report, 2004.

[21] ISO 22975-3:2014. Solar energy - collector components and materials - Part 3: Absorber surface durability.

[22] J. Bartl, M. Baranek. Emissivity of aluminium and its importance for radiometric measurement. Measurement Science Review V4, S3(2004) 31-36.

[23] Z. Chen, Q. Nguyen, T. Boström. Carbon Nanotube Spectrally Selective Solar Absorbers. EuroSun 2014 Conference Proceedings. DOI:10.18086/eurosun.2014.03.05.

[24] Y. Chiang, W. Lin, Y. Chang. The influence of treatment duration on multi-walled carbon nanotubes functionalized by H2SO4/HNO3 oxidation. Applied Surface Science 257 (2011) 2401 - 2410.

[25] F. Avile's, J.V. Cauich-Rodri'guez, L. Moo-Tah, A. May-Pat, R. Vargas-Coronado. Evaluation of mild acid oxidation treatments for MWCNT Functionalization. Carbon 47(2009) 2970-2975. 